Research article

\title{
Stratum corneum lipids liposomes for the topical delivery of 5- aminolevulinic acid in photodynamic therapy of skin cancer: preparation and in vitro permeation study Maria Bernadete R Pierre ${ }^{1}$, Antônio C Tedesco ${ }^{2}$, Juliana M Marchetti ${ }^{1}$ and M Vitória LB Bentley*1
}

Address: ${ }^{1}$ Faculdade de Ciências Farmacêuticas de Ribeirão Preto, Universidade de São Paulo, Av. do Café s/n, CEP 14040-903, Ribeirão Preto, SP, Brasil and ${ }^{2}$ Faculdade de Filosofia, Ciências e Letras de Ribeirão Preto, Universidade de São Paulo, Brasil

E-mail: Maria Bernadete R Pierre - mbrpierre@fcfrp.usp.br; Antônio C Tedesco - tedesco@ffclrp.usp.br; Juliana M Marchetti - jmarchet@usp.br; M Vitória LB Bentley* - vbentley@usp.br

*Corresponding author

This article is available from: http://www.biomedcentral.com/I47I-5945/I/5

(C) 200 I Pierre et al; licensee BioMed Central Ltd. Verbatim copying and redistribution of this article are permitted in any medium for any non-commercial purpose, provided this notice is preserved along with the article's original URL. For commercial use, contact info@biomedcentral.com

\begin{abstract}
Background: Photodynamic therapy (PDT) using 5-aminolevulinic acid (5-ALA) is a skin cancer therapy that still has limitations due to the low penetration of this drug into the skin. We have proposed in this work a delivery system for 5-ALA based on liposomes having lipid composition similar to the mammalian stratum corneum (SCLLs) in order to optimize its skin delivery in Photodynamic Therapy (PDT) of skin cancers.

Methods: SCLLs were obtained by reverse phase evaporation technique and size distribution of the vesicles was determinated by photon correlation spectroscopy. In vitro permeation profile was characterized using hairless mouse skin mounted in modified Franz diffusion cell.

Results: Size exclusion chromatography on gel filtration confirmed vesicle formation. SCLLs obtained by presented a degree of encapsulation of 5-ALA around 5.7\%. A distribution of vesicle size centering at around $500 \mathrm{~nm}$ and $400 \mathrm{~nm}$ respectively for SCLLs and SCLLs containing 5-ALA was found. In vitro 5-ALA permeation study showed that SCLLs preparations presented higher skin retention significantly $(p<0.05)$ on the epidermis without $S C+$ dermis, with a decreasing of skin permeation compared to aqueous solution.

Conclusions: The in vitro delivery performance provided by SCLLs lead to consider this systems adequate for the 5-ALA-PDT of skin cancer, since SCLLs have delivered 5-ALA to the target skin layers (viable epidermis + dermis) to be treated by topical PDT of skin cancer.
\end{abstract}

\section{Background}

Photodynamic therapy (PDT) is a modality of experimental cancer treatment using a photosensitizer, which localizes in tumor tissue and can produce tissue destruction upon absorption of light of an appropriate wave- length and dose [1]. The topical application of $5^{-}$ aminolevulinic acid (5-ALA) followed by light irradiation is a new concept of PDT [2]. 5-ALA is a prodrug that can be converted in situ into a highly fluorescent substance, protoporphyrin IX (PpIX), an effective photosensitizer 
by the heme biosynthetic pathway. The presence of exogenous 5-ALA bypasses feedback control, and thus may induce intracellular accumulation of photosensitizing concentrations of PpIX [3]. Topical photodynamic therapy using 5 aminolevulinic acid (5-ALA-PDT) has shown to be highly efficient for the topical treatment of a variety of superficial skin malignances [4]; it presents advantages over conventional treatments, like surgery, electrodessication, cryosurgery, topical application of podophylin or 5-fluorouracil, and radiotherapy [5]. 5ALA is a hydrophilic molecule and does not easily penetrate through intact skin or cell membranes. In order to improve the efficacy of ALA-PDT, particularly in thicker lesions, a number of options have been investigated. Penetration enhancers have been used to improve skin distribution of 5-ALA into the skin [6] and iron chelators [7] have been added to enhance the production of photosensitizer PpIX. 5-ALA application and light exposure protocols have been proposed [8]. Iontophoresis [9] and ultrasound [10] have been studied as physical methods to increase skin penetration of 5-ALA. In both case, enhanced production of PpIX in the skin was observed. 5ALA ester derivatives which present more lipophilic properties have been synthesized, higher PpIX level in different cell cultures having been found for these compounds compared to 5-ALA itself [11].

Delivery systems that can improve the tissue penetration and retention of 5-ALA lead to a desirable accumulation of PpIX in the skin, optimizing the PDT in this way. Lipids present in human stratum corneum (SC) are ceramides, cholesterol, free fatty acids and cholesteryl sulfate; phospholipids are absent [12]. These lipids are termed "skin lipids" and are arranged in a bilayer sheet structures filling the intercellular space in the SC. In the topical administration of drugs, this barrier must be overcome in order to provide sufficient drug amounts at the target tissue. The properties of human SC lipids are of specific interest in this context [13]. One of the first experimental approaches involved the examination of model lipid liposomes, the so-called SCLLs made from commercial lipids. SCLLs have been proposed as a topical drug carrier system [14] because their lipid composition is similar to SC lipids, which enables these systems to penetrate the epidermal barrier to a greater extent and act as "drug localizers" [15]. The main objective of the present work is to propose a topical delivery system for 5-ALA, based on SCLLs and to evaluate its in vitro skin release profile from these preparations.

\section{Materials and methods Materials}

5-ALA-HCl, bovine ceramide (Type III), cholesterol, palmitic acid and colesteryl sulfate were purchased from
Sigma. All other chemicals were analytical or HPLC grade.

\section{Preparation of SCLLs by the reverse phase evaporation technique}

Individual lipids were combined to obtain $10 \mathrm{mg}$ of a mixture containing lipids at the following concentrations (w/w): ceramide (50\%), cholesterol (28\%), palmitic acid $(17 \%)$ and colesteryl sulfate (5\%) in close approximation of the composition of SC lipids [12]. One mol \% $\alpha$-tocoferol was added to this formulations, as an antioxidant. Large unilamelar liposomes were prepared by reverse phase evaporation [16]. The lipidic mixture was dissolved in a mixture of diethyl ether: chloroform (1:1 volume ratio) to a volume of $50 \mathrm{~mL}$ in appropriate flask, and sonicated for $20-30 \mathrm{~min}$, The temperature was maintained between 5 and $10^{\circ} \mathrm{C}$ using a ice-bath. After complete dissolution of the lipidic mixture, the aqueous phase (sodium acetate $100 \mathrm{mM}$ buffer, $\mathrm{pH}$ 5.0) containing 5-ALA to yield a ratio of drug: lipids 1:3 was added. The resulting two-phase system was sonicated for 20-30 min in a bath-type sonicator and was placed on a rotator evaporator at $2 \mathrm{O}-25^{\circ} \mathrm{C}$ to remove the organic solvent under reduced pressure. The preparation was then heated at $70^{\circ} \mathrm{C}$ (using a thermo barrel attached to a circulating water-bath); this temperature is above to gel-liquid crystal transition temperature of the hydrated SC lipids (Tc $=$ $65^{\circ} \mathrm{C}$ ). The preparation containing $5 \mathrm{mg}$ lipid $/ \mathrm{mL}$ was then extruded using a stainless steel extrusion device ( $\mathrm{Li}-$ pex Biomembranes, Vancouver, BC, Canada) at $70^{\circ} \mathrm{C}$ through polycarbonate filters (Nucleopore Corporation, Pleasaton, Calif, USA) to an initial pore size of $5 \mu \mathrm{m}$ and subsequently of $1 \mu \mathrm{m}$.

\section{Characterization of SCLLs by size exclusion chromatogra- phy}

SCLLs preparations were subjected to molecular sieve chromatography on a Sephadex G-75 column $(15 \mathrm{~mm} \times$ $670 \mathrm{~mm}$ ) at room temperature, and eluted with a buffered sodium acetate (100 mM, pH 5.0) solution. Fraction collector, at a flow rate of $0.3 \mathrm{~mL} / \mathrm{min}$ collected the effluents. Absorbance measurements at $246 \mathrm{~nm}$ were done in the collected fractions in order to determine the elution profile of the SCLLs. 5-ALA was assayed in the collected fractions by the fluorimetric assay [17]. Prior to the 5ALA assay, $250 \mu \mathrm{L}$ ethanol was added to the liposomal fractions for the vesicles solubilization.

\section{Analysis of SCLLs vesicle average size by Dynamic Light Scattering}

Average particle size and size distribution of SCLLs were measured by photon correlation spectroscopy with a quasi-elastic laser light scattering (QELS) instrument (laser source $\mathrm{He}-\mathrm{Ne}, 633 \mathrm{~nm}$ and $90^{\circ}$ scattering angle). Vesicle size distribution and the polydispersity index 
(PI) were determined with dynamic light scattering using a photon correlating spectrometer (SYSTEM 4700Malvern). Samples were adjusted to the appropriate concentration range with sodium acetate buffer $(100 \mathrm{mM}$, $\mathrm{pH}$ 5.0). Measurements were made at $25^{\circ} \mathrm{C}$ and scattering angle of $90^{\circ}$. Cumulative analysis of scattering data was used to estimate effective diameters and polydispersity.

\section{In vitro permeation and retention studies}

Dorsal full-thickness skins of four weeks old male hairless mice (strain HRS/J, Jackson Laboratories, Bar Harbor, ME), were excised and immediately mounted in a modified Franz diffusion cell, kept at $37^{\circ} \mathrm{C}$. The donor medium was $0.5 \mathrm{~mL}$, either a SCLLs preparations or aqueous solution containing the same concentration of 5-ALA. Isotonic $\mathrm{pH} 5.0$ acetate buffer containing formalin $0.01 \%(w / w)$ was used as the acceptor medium, from which samples were collected at regular intervals during 36 hs. Flux values were obtained from steady state portion of the curve.

For skin retention study in vitro permeation experiments were performed as described above and the skins were removed from the diffusion cell after $36 \mathrm{~h}$, cleaned with cotton soaked in isotonic acetate buffer $(100 \mathrm{mM}, \mathrm{pH}$ 5.0), and then gently dried by pressing between two tissue papers. SC layers were removed by tape stripping method, using Scotch Crystal Tape ( $3 \mathrm{M}^{\mathrm{TM}}$, France). About 10 tape-strips were necessary to remove the SC from the skin samples. 5-ALA present in these tapestrips was extracted with isotonic acetate buffer. The remaining tissue (rest of epidermis + dermis) was homogenized in $10 \mathrm{~mL}$ of buffer for extraction of 5-ALA and filtered. 5-ALA amounts presented in the acceptor medium samples and tape strips and epidermis without SC + dermis extraction solutions were determined by fluorimetric assay [17]. The $\mathrm{pH} 5.0$ was used in the experiments because the higher 5-ALA stability in this $\mathrm{pH}$.

\section{Results}

Figure 1 shows the elution profile of SCLLs obtained by the phase reverse evaporation technique followed by extrusion, as well as elution of 5-ALA. The extent of drug entrapped in liposome vesicles can be determined by column chromatography or by other methods, followed by drug assay. Liposome preparations are a mixture of encapsulated and unencapsulated drug fractions. The unencapsulated drug fraction is also referred to as "free" drug. Free 5-ALA was eluted in the fractions $15-20$, distant from the void volume (Figure $1 \mathrm{~b}$ ). The elution profile of SCLLs presented by a single peak, close to the column's void; 5-ALA was also eluted in a unique peak, distant from the column's void. For the SCLLs samples prepared with 5-ALA at 1:3 drug:lipids ratio, a degree of encapsulation around $5.7( \pm 0.17) \% \mathrm{w} / \mathrm{v}$ was found. The
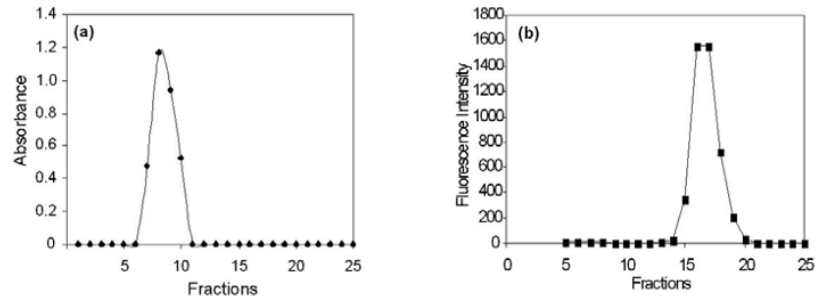

Figure I

Elution profile of: (a) SCLLs and (b) 5-ALA solutions using size exclusion chromatography on a Sephadex G-75 column.

vesicle size distribution determined by photon correlation spectroscopy has showed that SCLLs presented an average particle size of around $500 \mathrm{~nm}$, in accordance with the size vesicle obtained by the reverse evaporation technique [16]. In the presence of 5-ALA, it size was decreased to $400 \mathrm{~nm}$ (Table 1), indicating possible drugvesicle interactions. Figure 2 represents the in vitro permeation profile of 5-ALA from SCLLs and aqueous solution. 5-ALA flux through hairless mouse skin, calculated after reaching a steady state, was lower for SCLLs formulations ( $4.2 \pm 0.2 \mu \mathrm{g} / \mathrm{cm}^{2}$.h) than for aqueous solutions $\left(38.3 \pm 2.4 \mu \mathrm{g} / \mathrm{cm}^{2} . \mathrm{h}\right)$. The presence of SCLLS decreased the amount of 5-ALA transported through hairless mouse skin, after 36 hours to $500.9( \pm 32.5) \mu \mathrm{g}$, a value considerably significantly $(P<0.001)$ lower than 3681.0 $( \pm 104.65) \mu \mathrm{g}$ observed for aqueous solutions. However, the skin retention in the skin layers was increased by SCLLs formulation significantly $(P<0.05)$ only in the epidermis without $\mathrm{SC}+$ dermis in comparison with values for aqueous solution (Figure 3 ).

\section{Discussion}

The obtainment of a liposomal system with a greater interaction with the cutaneous tissue based on the similarity between its lipid composition and that of the SC may be an adequate strategy for improvement of 5-ALA skin delivery. Using exclusion chromatography it was possible to verify the presence of supramolecular aggregates in the SCLLs preparations obtained in the present study. SCLLs fractions were eluted with the void volume of the column and consisted of particles fully excluded from the internal volume of the gel ( 7 to 10 fractions; as shown in the Figure 1(a). Free 5-ALA appeared in the fractions 15 to 20 , as illustrated in the Figure 1(b). Size exclusion chromatography of SCLLs showed a single peak and vesicle formation; the individual lipids and smaller vesicles would elute in fractions distributed over the internal volume of the gel, distant from the void volume. The liposome fractions collected from the gel filtration column showed a low degree of about $5.7( \pm 0.17) \% \mathrm{w} / \mathrm{v}$ of 5 -ALA encapsulation. This low percentage of encapsulation 


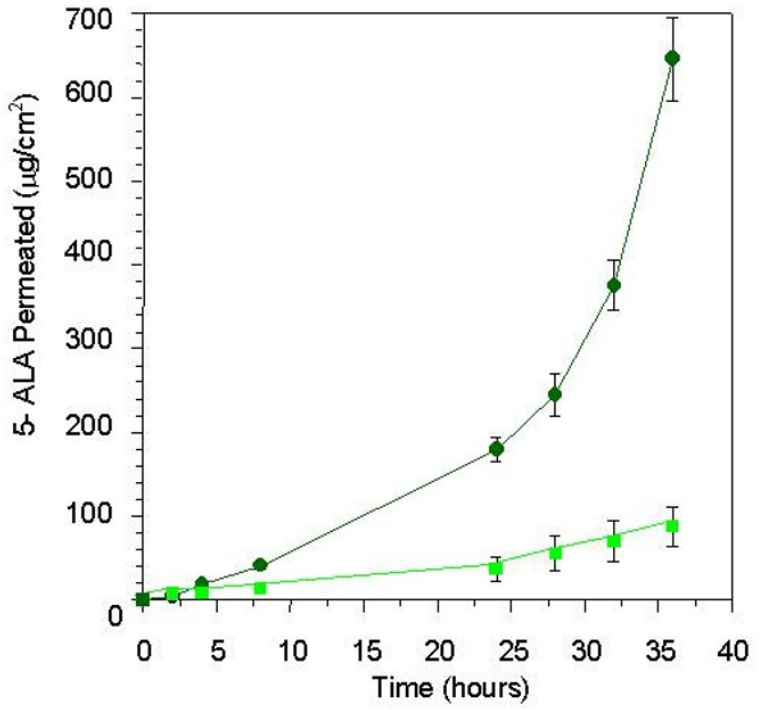

\section{Figure 2}

Amount of 5-ALA permeated $\left(\mu \mathrm{g} / \mathrm{cm}^{2}\right)$ through hairless mouse skin from the 5-ALA aqueous solution (black star) and SCLLs (square), as a function of time (hours).

may be due to the fact that 5-ALA is a small, hydrophilic molecule, probably capable of leaking out of the vesicles. In the presence of 5-ALA, a significant decrease $(P<$ 0.05 ) of the vesicle size to around $400 \mathrm{~nm}$ was observed, possibly characterizing an interaction at the molecular level between the drug and the lipids (Table 1).

Table I: Values of SCLLs sizes and their polidispersity using photon correlation spectroscopy ${ }^{*}$

\begin{tabular}{lll}
\hline Preparation & Mean size $(\mathrm{nm})$ & P.I.** $^{* *}$ \\
\hline & & \\
SCLLs alone & 500.0 & 0.570 \\
SCLLs containig 5-ALA & 400.1 & 0.570 \\
$\quad$ (drug:lipids, I:3) & &
\end{tabular}

\footnotetext{
* Scattering angle of the laser beam was $90^{\circ}$; temperature $25^{\circ} \mathrm{C}$, viscosity 0.890 and refraction index 1.33 . ${ }^{* *}$ Polidispersity index, meaning the measurement of the homogeneity of a dispersion, ranging from 0.0 (monodisperse) to I.0 (very heterogeneous).
}

In vitro permeation and retention studies showed that 5ALA in the liposomal system presented a lower permeation through mouse skin (Figure 2). Amazedly, it was observed a significant $(P<0.05)$ increase in the 5-ALA retention in epidermis without $\mathrm{SC}+$ dermis compared to aqueous solution (Figure 3). This behaviour can be explained as a probable consequence of SCLLs-skin interaction, providing a deposit effect for 5-ALA in the animal skin. In fact, liposomes have actually been referred to skin "drug localizers" [15]. Partition and diffusion in the SC lipids bilayers are important steps to delivery drugs to the subsequent skin layers (viable epidermis and dermis) [18], were most of skin cancer lesions takes place. In the present work, SCLLS containing 5-ALA showed adequate retention of the drug in the epidermis without SC + dermis, with decreased transdermal delivery (Figure 3). This behaviour shows that the liposomal system based on lipid composition similar to SC lipids provided a target delivery of 5-ALA into skin tissue to be treated in the topical PDT of skin cancer. Despite the low degree of 5-ALA encapsulation in SCLLs, a suitable 5-ALA delivery into skin, as well as its slower rate of permeation were observed. These properties are interesting to ensure PpIX formation over an adequate time period and amount. Our previous work [19] showed that lecithin in poloxamer gels can increase skin retention of hidrocortisone acetate into hairless mouse skin; they attributed this results to the increased lipid content of the SC caused by the phospholipid, in special within the SC intercellular region. Similarly to SC, SCLLs have ceramides (50\%) as the major lipid. This property may increase formulation-SC interactions faciliting thus, the partition of 5-ALA into the skin. The higher amount of 5ALA in the epidermis without SC + dermis can be explained because 5 -ALA is polar and charged. It can be partitioned promptly to the aqueous skin layers, despite SCLLs formulation is retained in the SC. For lipophilic drugs, it could find a higher retention in the SC, since SCLLs provide a favorable environment for these drugs. Other authors [20] have found by an in vivo stripping technique, that SCLLs showed a high affinity for SC indicated by a high amount of a fluorescent marker (sodium fluorescein), and suggesting a greater reservoir capacity of SC compared to similar lipid composition formulation (not a liposomal system). Liposome formulation based on phospholipids can interact with SC destabilizing, and fusing or mixing with the lipid matrix, acting as permeation enhancers and increasing the transdermal delivery [21]. SCLLs effect found in the present work is desirable for topical drug delivery [22], in special for skin cancer treatment based on 5-ALA PDT, in which a high 5-ALA skin retention may lead to greater PpIX accumulation into the skin. Since the success of this therapy is related to the amount of PpIX formed in the target tissue (viable epidermis and dermis), more efficient therapeutic effect may be expected from its utilization.

\section{Conclusions}

Liposomal preparations obtained using commercial lipids; the so-called SCLLs appear to be a useful 5-ALA de- 


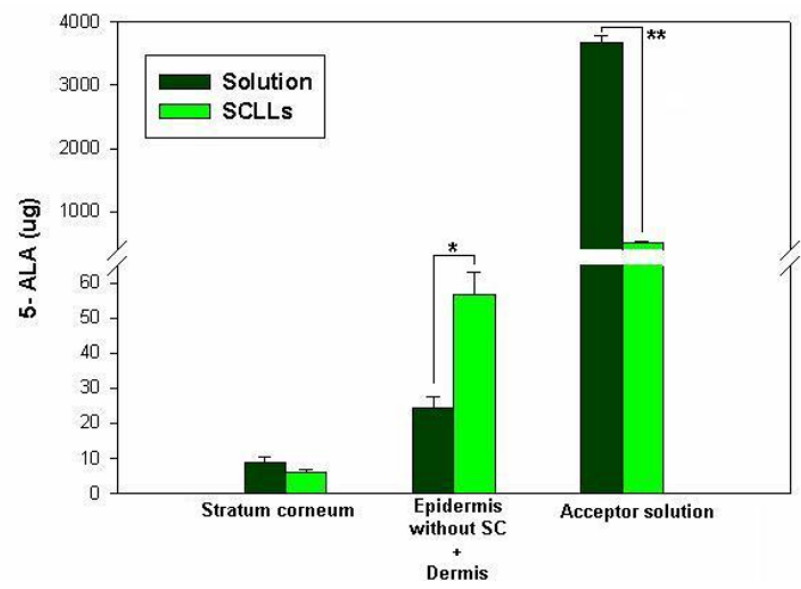

Figure 3

Amount of 5-ALA ( $\mu \mathrm{g})$ permeated and retained in the SC and epidermis without SC + dermis after 36 hours of in vitro permeation study. Errors bars indicate standard deviation of the mean. Statistical significance indicated by one-tailed Student's $t$ test: $\left.\left(^{*}\right) P<0.05\right)$ and $\left(^{* *}\right) P<0.001$.

livery system for topical PDT in skin cancers. Considering that adequate skin bioavailability of 5-ALA is important for this therapy, SCLLs preparations provided a good performance, by a slower permeation and delivery of the drug into epidermis without SC +dermis compared to aqueous solutions usually applied in 5ALA-PDT clinical procedure. A lipid bilayer structure, similar to SC lipid matrix is another advantage of SCLLs in favor of their interaction with the skin. Other authors [23] have found that these preparations permit a higher turnover of the skin barrier in comparison to a simple lipid mixture. Delivery systems that increase 5-ALA concentration in the target skin layers may improve the efficacy of topical PDT with this drug, since the PpIX accumulation is directly related to the presence of 5-ALA in the skin [1]. The encapsulation problem found in the present work may in part be solved by the use of ester derivatives of 5-ALA [11], which present higher molecular weight and lipophilic characteristics than 5-ALA itself, which can provide higher encapsulation degree and infer in improved retention into the target tissue with consequently improvement of PpIX accumulation.

The results obtained in this work, indicate that SCLLs formulations appear to be suitable for topical delivery of 5-ALA. Efficiency of encapsulation, stability of SCLLs containing 5-ALA or its ester derivatives, skin permeation profiles as well as in vivo PpIX production in treated skin, remain to be optimized in order to fill the gap in knowledge about drug delivery systems to be used in PDT.

\section{Competing Interests}

None declared.

\section{Acknowledgements}

This work was supported by Fundação de Amparo à Pesquisa do Estado de São Paulo (FAPESP), Brazil. We would like to thank Dr. Pietro Ciacaglini for the assistance on chromatography studies. We are also thankful to Dr. A. $M$. Rothschild for revision of this manuscript.

\section{References}

I. De Rosa FS, Bentley MVLB: Photodynamic therapy of skin cancers: sensitizers, clinical studies and future. Pharm. Res. 2000 , I 7: | 447-| 455

2. Fuchs J, Thiele J: The role of oxygen in cutaneous photodynamic therapy. Free Radical Biology \& Medicine 1998, 24:835-847

3. Ackermann G, Abels C, Baumler W, Langer S, Landthaler M, Lang EW, Szeimies RM: Simulations on the selectivity of 5-aminolevulinic acid-induced fluorescence in vivo. J. Photochem. Photobiol. B: Biol. 1998, 47:121-128

4. Robinson DJ, Bruijn HS, Van Der Veen N, Stringer MR, Brown SB, Star WM: Fluorescence photobleaching of ALA-induced protoporphyrin IX during photodynamic therapy of normal hairless mouse skin: the effect of light dose and irradiance and the resulting biological effect. Photochem. Photobiol. 1998, 67: $140-149$

5. Morton AC, Whitehurst C, Moseley H, Mccoll JH, Moore JV, Mackie $\mathrm{R}$ : Comparision of photodynamic therapy with cryotherapy in the treatment of Bowen's disease. Br. J. Dermatology 1996, | 35:766-77|

6. De Rosa FS, Marchetti JM, Thomazini JA, Tedesco AC, Bentley MVLB: A vehicle for photodynamic therapy of skin cancer: influence of dimethylsulphoxide on 5-aminolevulinic acid in vitro cutaneous permeation and in vivo protoporphyrin IX accumulation determined by confocal microscopy. J.Control. Release 2000 , 65:359-366

7. Malik Z, Kostenich L, Roitman B, Ehrenberg B, Orestein A: Topical appication of 5-aminolevulinic acid, DMSO and EDTA: protoporphyrin IX accumulation in skin and tumours of mice. J. Photochem. Photobiol.B: Biol 1995, 28:213-218

8. Kalka K, Merck H, Mukhtar H: Photodynamic therapy in dermatology. J. Am. Acad. Dermatol 2000, 42:389-4I3

9. Lopez RFV, Bentley MVLB, Delgado-Charro MB, Guy RH: Iontophoretic delivery of 5-aminolevulinic acid (ALA): effect of pH. Proceed. Int'l. Symp. Control. Rel. Bioact. Mater. 2000, 27:7407

10. MA L, Moan J, Peng Q, lani V: Production of protoporphyrin IX induced by 5 -aminolevulinic acid in transplanted human coloadenocarcinoma of nude mice ca be increased by ultrasound. Int. J. Cancer I 998, 78:464-469

II. Kloek J, Akkermans W, Van Henegouwen GMJB: Derivatives of 5aminolevulinic acid for photodynamic therapy: enzimatic conversion into protoporphyrin. Photochem. Photobiol. 1998, 67:150-154

12. Downing DT, Abraham W, Wegner BK, Willman KW, Marshall JL: Partition of dodecyl sulfate into stratum corneum lipid liposomes. Arch. Dermatol. Res. 1993, 285:151-I57

13. Korting HC, Stolz W, Schmid MH, Maierhofer G: Interaction of liposomes with human epidermis reconstructed in vitro. $\mathrm{Br}$. J. Dermatol 1995, | 32:57|-579

14. Wertz PW, Abraham W, Landmann L, Downing DT: Preparation of liposomes from stratum corneum lipids. J. Invest. Dermatol I986, 87:582-584

15. Schmid $\mathrm{MH}$, Korting HC: Therapeutic progress with topical liposome drugs for skin disease. Adv. Drug Deliv. Rev. 1996, I 8:335342

16. Szocka F, Papahadjopoulos D: Procedure for preparation of liposomes with large internal aqueous space and high capture by reverse-phase evaporation. Proc. Natl. Acad. Sci. 1978, 75:41944198

17. Oishi H, Nomiyama H, Nomiyama K, Tomokuni K: Fluorometric HPLC determination of 5-aminolevulinic acid (ALA) in the plasma and urine of lead workers: biological indicators of lead exposure. J. Anal. Toxicol. 1996, 20:106-I I0 
18. Mitragotri S: In situ determination of partition and diffusion coefficients in the lipid bilayers of stratum corneum. Pharm. Res 2000, 17:1026-1029

19. Bentley MVLB, Kedor ERM, Vianna RF, Collet JH: The influence of lecithin and urea on the in vitro permeation of hydrocortisone acetate through skin from hairless mouse. Int. J. Pharm 1 997, 1 46:255-262

20. Coderch L, De Pera M, Perez-Cullell N, Estelrich J, De La Maza, Parra $\mathrm{JH}$ : The effect of liposomes on skin barrier structure. Skin Pharmacol. Appl. Skin Physiol 1999, I 2:235-246

21. Kirjavainen M, Urtti A, Jaaskelaine I, Suhonnen TM, Paronen P, Valjakka-Koskela R, Kiesvaaro J, Monkkonen J: Interaction of liposomes with human skin in vitro - the influence of lipid composition and structure. Biochim. Biophys. Acta 1996, I304:179-189

22. Shah VP, Behl CR, Flynn GL, Higuchi WI, Schaefer H: Principles and criteria in the development and optimization of topical therapeutical products. Int. J. Pharm. 1992, 82:21-28

23. Hatziantoniou S, rallis S, Demetzos C, Papaionnou GT: Pharmacological activity of natural lipids on skin barrier disruption model. Pharmacol. Res 2000, 42:55-59

Publish with BioMed Central and every scientist can read your work free of charge

"BioMedcentral will be the most significant development for disseminating the results of biomedical research in our lifetime."

Paul Nurse, Director-General, Imperial Cancer Research Fund

Publish with BMC and your research papers will be:

- available free of charge to the entire biomedical community

- peer reviewed and published immediately upon acceptance

- cited in PubMed and archived on PubMed Central

- yours - you keep the copyright 